\section{Ovine psittacosis and sarcoidosis in a pregnant woman}

\author{
Barbara A Crosse, P Gomes, M M Muers
}

\begin{abstract}
A woman in the first trimester of pregnancy presented with pneumonia and hilar lymphadenopathy after exposure to lambing ewes. She subsequently aborted. Infection with Chlamydia psittaci of ovine origin was confirmed. Pregnant women are susceptible to this infection, which may cause life threatening disease. The patient also had features of sarcoidosis, and the two conditions ran a similar time course. There is a possibility that ovine psittacosis caused an illness indistinguishable from sarcoidosis.
\end{abstract}

Chlamydia psittaci of ovine origin causes enzootic abortion of ewes. People may acquire the infection from sheep, particularly during the lambing season. The agent may cause severe multisystem disease in pregnant women, usually during the second and third trimester, with appreciable mortality for both mothers and fetuses. ${ }^{1}$ Mild infections are also recognised. ${ }^{2}$ Pneumonia rarely predominates, in contrast to infection with $C$ psittaci of avian origin. The precise incidence of the disease in man is unknown. Since 1985 less than $2 \%$ of cases of serologically proved $C$ psittaci infection have been attributable to the ovine strain on epidemiological grounds, according to the Public Health Laboratory Service communicable disease surveillance centre (S Young, personal communication). We describe a woman who was infected with $C$ psittaci of ovine origin during the first trimester of pregnancy, and who presented with pneumonia and spontaneous abortion. She also had clinical features compatible with a diagnosis of sarcoidosis.

Infectious Diseases

Unit, Seacroft

Hospital, Leeds

LS14 6UH

B A Crosse

Department

of Respiratory

Medicine, Killingbeck

Hospital, Leeds LS14

6UQ

P Gomes

M M Muers

Reprint requests to:

Dr Crosse

Accepted 26 April 1991

Case report

A 32 year old previously fit woman presented after five weeks of fever and malaise, two weeks of cough producing purulent sputum, and two days of left sided pleurisy. She was six weeks' pregnant. One week before the onset of the illness she had visited a farm where ewes were lambing. She had not handled the animals. There was no history of contact with birds or with any patients with psittacosis.
On examination she was afebrile, and remained so throughout. She had bilateral basal inspiratory crackles. Initial investigations showed a total white cell count of $6 \cdot 2 \times$ $10^{9} / 1$ (77\% neutrophils), and plasma viscosity of $1.79 \mathrm{mPa}$. A chest radiograph showed right lower lobe consolidation, and hilar lymphadenopathy (figure). Non-bacterial pneumonia was diagnosed and she was given oral erythromycin. Haemophilus parainfluenzae was isolated from sputum. Despite treatment her cough and pleurisy continued. Underlying conditions such as sarcoidosis, lymphoma, and connective tissue disease were considered. The results of the following investigations were normal: serum concentrations of transaminase, albumin, calcium, immunoglobulins, and cold agglutinins; angiotensin converting enzyme activity; and DNA binding capacity; and autoantibodies and rheumatoid factor were absent. No antibodies to Brucella $\mathrm{sp}$, influenza A and B viruses, Mycoplasma pneumoniae, Chlamydia group B, adenovirus, Coxiella burnetti, cytomegalovirus, Legionella pneumophila, Epstein-Barr virus, or Toxoplasma gondii were detected. Sputum cultures were negative for acid fast bacilli, $M$ pneumoniae, and $L$ pneumophila. Fibreoptic bronchoscopy showed bronchial inflammation, confirmed histologically.

A newspaper article at this time described enzootic abortion in ewes at a farm neighbouring that visited by the patient. Paired serum samples after one week showed titres of complement fixing antibody to Chlamydia group B of $1 / 512$ and $1 / 256$, confirmed by indirect immunofluorescence. The initial serum specimen had shown prozoning in the complement fixation test, explaining the false negative result when the specimen was screened at a dilution of $1 / 32$. Infection with $C$ psittaci of ovine origin was confirmed by the presence of IgM specific for $C$ psittaci of ovine origin at a titre of $1 / 2560$. Enzootic abortion due to $C$ psittaci was confirmed in the sheep flock on the farm visited by the patient.

Fourteen days after admission the patient had a spontaneous abortion. Uterine curettings were cultured for chlamydia, but were sterile. She was given oral oxytetracycline and recovered symptomatically over two months. Despite resolution of the consolidation on the chest radiograph hilar lymphadenopathy persisted. A Kveim test seven weeks after the onset of illness showed granulomas consistent with sarcoidosis in the absence of any foreign body. The serum angiotensin converting enzyme concentration two months after presentation was raised at $33.3 \mathrm{U} / 1$. Over the following eight months the concentration was raised on three further occasions and a diagnosis of sarcoidosis was accepted.

The patient was reviewed over 12 months and remained well. The hilar lymphadenopathy resolved and the serum angiotensin 


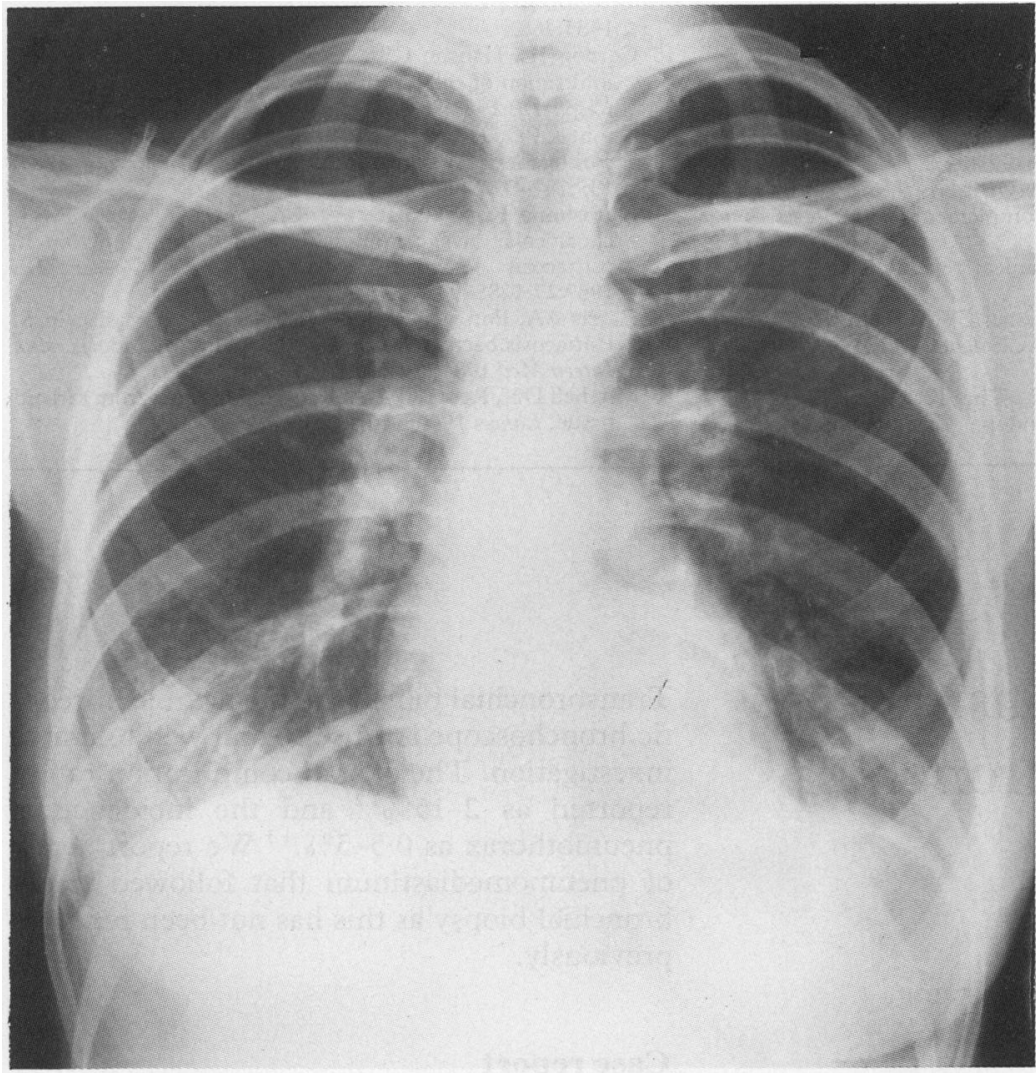

Chest radiograph of a pregnant woman infected with Chlamydia psittaci of ovine origin, who also had sarcoidosis, showing pulmonary consolidation and hilar lymphadenopathy.

converting enzyme activity fell to normal. The titre of complement fixating antibody to $C$ psittaci fell to $1 / 16$, and IgM specific for the ovine strain of $C$ psittaci fell to less than $1 / 80$. Clinical resolution of both the infection and sarcoidosis were therefore confirmed.

\section{Discussion}

Infection with the ovine strain of $C$ psittaci is endemic in the sheep population of the United Kingdom and causes abortion, stillbirth, and the birth of weak lambs. ${ }^{3}$ The organism differs in its DNA composition from other strains of $C$ psittaci that may infect animals and birdsfor example, the avian strain and the strains causing feline conjunctivitis and. mouse pneumonitis. ${ }^{4}$ Humans may acquire ovine psittacosis through contact with infected ewes. Laboratory acquired infection also occurs. ${ }^{25}$ Most cases reported have occurred in pregnant women in contact with lambing flocks, who have contracted the infection in the second or third trimester of pregnancy and have presented with a fulminant multisystem infection with a high mortality for the fetuses and occasionally with death of the mother. ${ }^{16-8} C$ psittaci was isolated from the placenta in one instance. ${ }^{8}$ A case reported in the first trimester of pregnancy was associated with pneumonia and jaundice. ${ }^{9}$ Uncomplicated pneumonia occurred in a non-pregnant laboratory worker in the first documented laboratory acquired infection, ${ }^{4}$ and mild respiratory tract infection was reported in a laboratory outbreak. ${ }^{2}$ The case we report therefore differs from most in that it occurred in the first trimester of pregnancy and was manifested by uncomplicated pneumonia and abortion. Women at all stages of pregnancy may therefore be at risk of infection, and should avoid contact with sheep, particularly at lambing time.

The case presented is also unusual in that there were clinical and pathological features of sarcoidosis. Some reports claim a high incidence of hilar lymphadenopathy with pneumonia due to $C$ psittaci (avian origin), ${ }^{10}$ but other large series do not. ${ }^{11}$ The latter view is confirmed by our own experience, ${ }^{12}$ justifying our search for an alternative diagnosis. Serum angiotensin converting enzyme activity is raised in about $86 \%$ of biopsy proved cases of sarcoidosis, but may also be raised in infections such as coccidioidomycosis and tuberculosis. ${ }^{13}$ The Kveim reaction of the biopsy specimen was believed to be a true positive in this case, though the reaction is considered selective rather than specific for sarcoidosis. ${ }^{14}$ The diagnosis of sarcoidosis is best made by detecting non-caseating granuloma in biopsy material from affected organs but this was not considered justifiable in this case. $C$ psittaci infection may also stimulate the formation of granulomas, particularly in the liver. ${ }^{15}$ Two other features of sarcoidosis-namely, the presence of $T$ cells in bronchoalveolar lavage fluid and increased uptake of gallium in affected tissues ${ }^{16}$-have recently been noted in $C$ psittaci infection. ${ }^{17}$ In a case report $^{18}$ two siblings presented with features of sarcoidosis-fever, rash, hilar lymphadenopathy, and liver granulomas. In one $C$ psittaci was isolated from the blood, and there was a diagnostic rise in antibody titres. A parakeet was the source of infection. As the other sibling failed to show a diagnostic rise in antibodies familial sarcoidosis was diagnosed, though the time course of the illness was similar and this patient was also exposed to the parakeet. The possibility that sarcoidosis may be caused by an infectious agent has been considered. ${ }^{19}$ The temporal association between the course of $C$ psittaci infection and the detection and resolution of sarcoidosis in our case raises the possibility that $C$ psittaci is capable of inducing a sarcoidosis like illness.

We thank Drs P J Stanley and J Stevenson for allowing us to report this case and Dr D Carrington, department of virology, Glasgow Royal Infirmary, for performing the serological tests for Glasgow Royal Infirmary, for
the ovine strain of $C$ psittaci.

1 Beer RJS, Bradford WP, Hart RJC. Pregnancy complicated by psittacosis acquired from sheep. $B M J 1982 ; 284$ 1156-7.

2 Baker CC, Cooper B. A case of good management. J Infect 1983;6:71-3.

3 Johnson FWA. Chlamydiosis. British Veterinary Journal 1983;139:93-101.

4 McClenaghan M, Herring AJ, Aitken ID. Comparison of Chlamydia psittaci isolates by DNA restriction endonuclease analysis. Infect Immun 1984;45:384-9.

5 Barwell CF. Laboratory infection of man with virus of enzootic abortion of ewes. Lancet 1955;269:1369-71.

6 Roberts W, Grist NR, Giroud P. Human abortion associated with infection by ovine abortion agent. BMJ 1967;iv:37.

7 McKinlay AW, White N, Buxton D, et al. Severe Chlamydia psittaci sepsis in pregnancy. $Q J$ Med 1985;222:689-96.

8 Helm CW. Smart GE, Cumming AD, et al. Sheep-acquired severe Chlamydia psittaci infection in pregnancy. Int $J$ Gynaecol Obstet 1989;28:369-72.

9 McGivern D, White R, Paul ID, et al. Concomittant zoonotic infection with ovine Chlamydia and $Q$ fever in 
pregnancy: clinical features, diagnosis, management and public health implications. Br J Obstet Gynaecol 1988; 95:294-8.

10 Stenstrom R, Jansson E, Wager O. Ornithosis pneumoni with special reference to roentengenological lung findings. Acta Med Scand 1962;171:349-56.

11 Jorgensen MB, Steffenson KA. Ornithosis. An analysis of 44 human cases with positive complement fixation tests. Dan Med Bull 1956;3:20-4.

12 Crosse BA. Psittacosis-a clinical review. J Infect 1990; 21:251-9.

13 Schultz T, Milre WC, Bedrossian CWM. Clinical application of measurement of ACE level. JAMA 1979;242: $439-41$.

14 Scadding JG, Mitchell DN. The Kveim reaction. In: Sarcoidosis. 2nd ed. London: Chapman and Hall,
1985:468.

15 Cornog JL, Hanson CW. Psittacosis as a cause of miliary infiltration of the lung and hepatic granulomas. Am Rev Respir Dis 1968;98:1033-9.

16 Thomas PD, Hunninghake GW. Current concepts of the pathogenesis of sarcoidosis. Am Rev Respir Dis 1987, 135:747-60.

17 Kurashima K, Watanabe A, Kawamura Y, et al. Bronchoalveolar lavage fluid findings and lung function in psittacosis [English abstract]. Jap J Thorac Dis 1989;27:1288-93.

18 Harris AA, Pottage JC, Kessler HA, Zeihen M, Levin S. Psittacosis bacteraemia in a patient with sarcoidosis. Ann Intern Med 1984;101:502-3.

19 Mitchell DN, Rees RJW. A tranmissible agent from sarcoid tissue. Lancet 1969;ii:81-2.

\section{Pneumomediastinum after a transbronchial biopsy}

\author{
Matthew Naughton, Lou Irving, \\ Allan McKenzie
}

Department of Thoracic Medicine M Naughton L Irving

Department of Diagnostic Radiology A McKenzie

Repatriation General Hospital, Heidelberg, Victoria 3081, Australia

Reprint requests to: Dr Naughton

Accepted 15 February 1991

\begin{abstract}
A pneumomediastinum occurred after a transbronchial biopsy in a woman with pulmonary fibrosis and was confirmed by computed tomography. Although pneumomediastinum has a pathogenesis similar to that of pneumothorax it has not been reported after transbronchial biopsy.
\end{abstract}

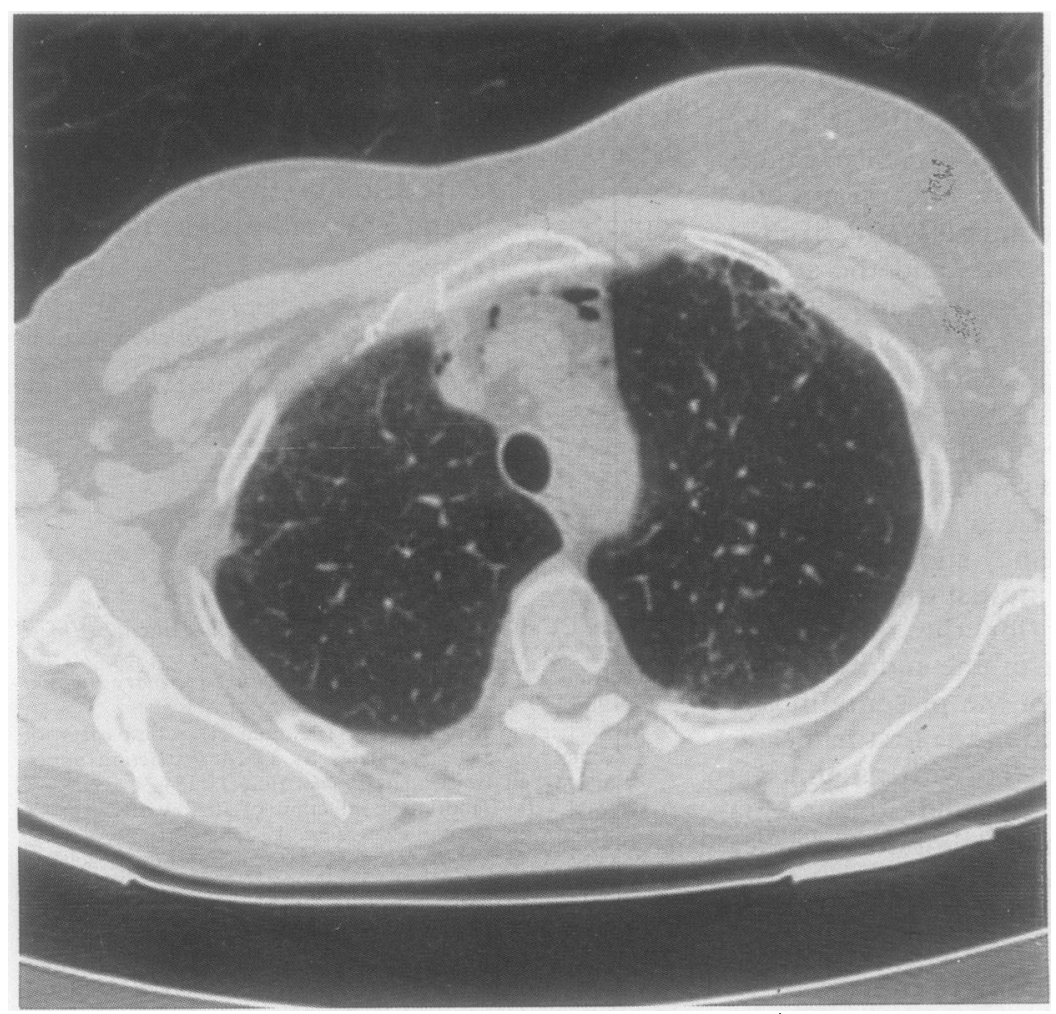

Computed tomogram of the chest showing fine reticular nodular pattern, a few small cysts, and gas in the anterior mediastinum.
Transbronchial biopsy via the flexible fibreoptic bronchoscope is a useful and well tolerated investigation. The overall complication rate is reported as $2-15 \%{ }^{12}$ and the incidence of pneumothorax as $0 \cdot 5-5 \% .{ }^{13} \mathrm{We}$ report a case of pneumomediastinum that followed transbronchial biopsy as this has not been reported previously.

\section{Case report}

A 53 year old housewife was admitted to hospital for investigation of a four week history of exertional dyspnoea, haemoptysis, and a bilateral pulmonary fine reticular nodular pattern on the chest radiograph. She had had non-erosive arthritis, malar rash, Raynaud's phenomenon, and the sicca syndrome for several years. Results of previous serological tests were positive for antinuclear factor (1/640, nucleolar pattern), suggesting systemic lupus erythematosus. She was an exsmoker and denied contact with tuberculosis. She had otherwise been in good health. Her medication was naproxen $250 \mathrm{mg}$ daily. The only abnormal finding of the clinical examination was pulmonary crepitations. There was no rash, clubbing, lymphadenopathy, or organomegaly.

Investigations, including sputum examination for acid fast bacilli and malignant cells, ventilation-perfusion lung scanning, urine and blood culture, serological testing for atypical pneumonia, examination of the clotting profile, and electrocardiography, gave normal results. Respiratory function tests showed that $\mathrm{FEV}_{1}$ was 2.51 (109\% predicted), vital capacity 2.91 ( $100 \%$ predicted), and transfer factor for carbon monoxide 4.6 $\mathrm{mmol} / \mathrm{min} / \mathrm{kPa}(61 \%$ predicted).

She underwent flexible fibreoptic bronchoscopy and transbronchial biopsy of the left lung under local anaesthesia with oxygen administered intranasally; she was monitored by electrocardiography and pulse oximetry. She was calm throughout the procedure and there was no excessive coughing. Fluoroscopy was used for the transbronchial biopsy. After the fourth biopsy specimen had been taken she suddenly developed severe substernal chest pain. There was no fall in oxygen saturation or change in cardiac rhythm or vital 